\title{
GUILHERME DE LA PENHA - UM CIENTISTA MULTIFACETADO
}

\section{GUILHERME DE LA PENHA - A SCIENTIST ALL PURPOSE}

\author{
Ana Paula Nascimento Pegado Couto ${ }^{1}$ \\ Universidade do Estado do Pará \\ Lucas Antônio Mendes de Lima ${ }^{2}$ \\ Universidade do Estado do Pará \\ Miguel Chaquiam ${ }^{3}$ \\ Universidade do Estado do Pará
}

\begin{abstract}
Resumo
Após pesquisa documental realizada em Belém do Pará, retratamos alguns passos do cientista paraense Guilherme Maurício Souza Marcos de La Penha, considerado por alguns como um dos maiores geradores e gerenciadores da ciência no Brasil. Nosso objetivo foi de reescrever a história de vida, formação acadêmica, atuação profissional e produção intelectual do multifacetado cientista paraense Guilherme de La Penha tendo em vista a divulgação de seus trabalhos no meio acadêmico e sociedade em geral. Citamos como suporte teórico os artigos aprovados em encontros nacionais e internacionais, também partes integrantes da tese de Miguel Chaquiam (2012), que completam os traços biográficos de Guilherme de La Penha. Apresentamos traços de Guilherme de La Penha, dentre eles biográficos e acadêmicos, iniciados em Belém do Pará, visando à construção de um perfil e a caracterização das diversas faces. Para tanto, percorremos parte da sua produção científica, os cargos de gestão por ele ocupados e, em especial, os textos escritos em comemoração ao bicentenário de morte do matemático Leonhard Euler. Os resultados desta pesquisa proporcionaram uma ampliação e consolidação das diversas faces de La Penha de modo que pudéssemos revelar as produções acadêmicas que, de certa forma, não se tornaram tão conhecidas ou divulgadas nos meios acadêmico e científico, com vista a torná-las públicas e acessíveis, assim como, retirá-lo do anonimato em que se encontra. Em decorrência deste trabalho é possível perceber a importância de se divulgar as obras desse e de outros cientistas que fazem parte da nossa história, bem como, preservar registros históricos e arquivos particulares de homens cujas biografias completam a história do nosso país.
\end{abstract}

Palavras-chave: História da Matemática; Cientista Paraense; Guilherme de La Penha.

\section{Abstract}

After desk research conducted in Belém do Pará, depict steps of Pará scientist

\footnotetext{
1 ana.couto16@hotmail.com.

2 lucasamlima@outlook.com.

3 miguelchaquiam@gmail.com.
} 
Guilherme Mauricio Souza Marcos de La Penha, considered by some as one of the biggest generators and science managers in Brazil. Our goal was to rewrite the life history, educational background, professional experience and intellectual production of the multifaceted scientist paraense Guilherme of La Peña with a view to disseminating their work in academia and society at large. We quote as theoretical support the articles approved in national and international meetings, also integral parts of the thesis Miguel Chaquiam (2012), which complete the biographical traits of Guilherme de La Penha. Have traces Guilherme of La Penha, including biographical and academic, started in Belém do Pará, in order to build a profile and characterization of the different faces. Therefore, we have come part of their scientific, management positions that he occupied and, in particular, the texts written in celebration of the mathematician's death bicentenary Leonhard Euler. The results of this research provide expansion and consolidation of the various faces of La Penha so we could reveal the academic productions that somehow did not become as well-known or disclosed in the academic and scientific resources with a view to making them public and accessible, as well as remove it from the anonymity that is. As a result of this work it is possible to realize the importance of disseminating the work of this and other scientists who are part of our history as well as preserving historical records and private archives men whose biographies complete the history of our country.

Keywords: History of Mathematics; Paraense scientist; Guilherme de La Penha.

\section{Introdução}

Atraídos inicialmente pelas discussões sobre o ensino da História da Matemática, matemáticos paraenses e do conhecimento da tese do professor Miguel Chaquiam (2012), vinculada ao Programa de Pós-Graduação em Educação da Universidade Federal do Rio Grande do Norte, sob orientação do professor Iran Abreu Mendes, desenvolveu-se esta pesquisa a partir dos trabalhos que influênciaram a relevância de Guilherme de La Penha, para a História da Matemática no estado do Pará.

Guilherme Mauricio Souza Marcos de La Penha, filho de Miguel Marcos de La Penha e Nair Souza Marcos de La Penha, natural de Belém (PA), cidade com que sempre manteve fortes laços, nascido em 09 de março de 1942. Em Belém desenvolveu sua formação básica, onde cursou os ciclos primário e ginasial no extinto Instituto Suíço Brasileiro e no Colégio Marista Nossa Senhora de Nazaré, respectivamente. O quadro a seguir retrata de modo global sua formação acadêmica:

Quadro 1 - Formação Acadêmica de Guilherme de La Penha 
Ana Paula Nascimento Pegado Couto, Lucas Antônio Mendes de Lima e Miguel Chaquiam GUILHERME DE LA PENHA - Um cientista multifacetado

\begin{tabular}{|c|c|c|}
\hline Ano & Formação & Instituição \\
\hline $1959-1960$ & Curso de Agrimensor & $\begin{array}{c}\text { Escola Técnica de } \\
\text { Agrimensura do Pará }\end{array}$ \\
\hline $1960-1964$ & Curso de Engenharia Mecânica & $\begin{array}{l}\text { Escola Politécnica da } \\
\text { PUC - Rio }\end{array}$ \\
\hline $1961-1963$ & $\begin{array}{c}\text { Curso de Aperfeiçoamento em } \\
\text { Matemática }\end{array}$ & $\begin{array}{l}\text { Instituto de Matemática } \\
\text { Pura e Aplicada }\end{array}$ \\
\hline $1964-1965$ & Mestrado em Engenharia Mecânica & PUC - Rio \\
\hline $1965-1966$ & $\begin{array}{l}\text { Mestrado em Matemática Aplicada e } \\
\text { Física Teórica (Bachelor of Arts) }\end{array}$ & $\begin{array}{l}\text { Universidade de Cambridge } \\
\text { (Inglaterra) }\end{array}$ \\
\hline $1966-1968$ & $\begin{array}{l}\text { Doutorado na área de Matemática } \\
\text { Aplicada e Mecânica dos Sólidos }\end{array}$ & $\begin{array}{l}\text { Universidade de Houston } \\
\text { (EUA) }\end{array}$ \\
\hline 1967 & Curso de Mecânica do Contínuo & $\begin{array}{c}\text { Universidade de Brown } \\
\text { (EUA) e no Instituto } \\
\text { politécnico da Virgínia (EUA) }\end{array}$ \\
\hline $1968-1969$ & $\begin{array}{l}\text { Pós-Doutorado em Matemática } \\
\text { Aplicada }\end{array}$ & $\begin{array}{c}\text { Universidade de Carnegie- } \\
\text { Mellon (EUA) }\end{array}$ \\
\hline
\end{tabular}

Fonte: Chaquiam (2012)

La Penha apresentou uma vasta quantidade de publicações em torno de sua carreira acadêmica, o autor produziu mais de 75 artigos de pesquisa, segundo Bassalo (1997), publicados em periódicos especializados nacionais e internacionais. Além das substanciais contribuições acima mencionadas, La Penha sempre se fez presente em cargos importantes conforme consta no Quadro 2:

Quadro 2 - Cargos ocupados por Guilherme de La Penha 
Ana Paula Nascimento Pegado Couto, Lucas Antônio Mendes de Lima e Miguel Chaquiam

GUILHERME DE LA PENHA - Um cientista multifacetado

\begin{tabular}{|c|l|}
\hline \multicolumn{1}{|c|}{ Ano } & \multicolumn{1}{c|}{ Cargo } \\
\hline $\mathbf{1 9 6 9}$ - 1977 & Diretor do Instituto de Matemática da UFRJ \\
\hline $\mathbf{1 9 7 7}$ - 1978 & Diretor de Desenvolvimento Científico da FINEP \\
\hline $\mathbf{1 9 7 9}$ - $\mathbf{1 9 8 0}$ & Secretário de Educação Superior do MEC \\
\hline $\mathbf{1 9 8 0}$ - 1982 & Vice-Presidente do CNPq \\
\hline $\mathbf{1 9 8 3}$ & Assessor Especial do CNPq para Assuntos da Amazônia (MPEG) \\
\hline $\mathbf{1 9 8 4}$ - 1985 & $\begin{array}{l}\text { Assistente Especial do Departamento de Ciência e } \\
\text { Tecnologia da OEA (EUA) }\end{array}$ \\
\hline $\mathbf{1 9 8 5}$ - 1986 & $\begin{array}{l}\text { Consultor do BID (EUA), Diretor do MPEG e } \\
\text { Secretário de Cultura do Estado do Pará }\end{array}$ \\
\hline $\mathbf{1 9 8 7}$ - 1991 & $\begin{array}{l}\text { Diretor do MPEG e Secretário de Ciência e } \\
\text { Tecnologia e Meio Ambiente }\end{array}$ \\
\hline $\mathbf{1 9 9 1}$ - 1994 & $\begin{array}{l}\text { Secretário de Cultura do Estado do Pará e } \\
\text { Presidente da Fundação Cultural Tancredo Neves, }\end{array}$ \\
\hline $\mathbf{1 9 9 5}$ - 1996 & $\begin{array}{l}\text { Diretor de Programas Espaciais da Secretária de } \\
\text { Assuntos Estratégicos da Agência Espacial Brasileira }\end{array}$ \\
\hline
\end{tabular}

Fonte: Chaquiam (2012)

Antes de completar 54 anos, no dia 6 de fevereiro de 1996 veio a falecer, quando ocupava o cargo de Diretor de Programas Espaciais da Secretária de Assuntos Estratégicos da Agência Espacial Brasileira, tornando-se uma grande perda para a vida acadêmica e administrativa de ciência no Brasil.

Embora tenha constituído um currículo invejável, observa-se no meio acadêmico que há certo desconhecimento do nome Guilherme de La Penha, principalmente nos cursos de Licenciatura Matemática em Belém do Pará. Enquanto alunos da graduação, observamos a ausência de trabalhos e de debates em eventos que abordam este tema. A exemplo, do Seminário de Cognição e Educação Matemática (SCEM), Encontro Paraense de Educação Matemática (EPAEM), semanas acadêmicas e outras discussões dentro das Universidades locais.

O trabalho resultou uma reconstrução da história sobre vida e obra de Guilherme de La Penha e, de acordo com Chaquiam (2012), este possuia o perfil de um intelectual 
múltiplo, cujo pensamento sobre ciência, tecnologia, formação de cientistas e educadores estiveram em harmonia nos seus escritos e sua prática profissional.

O trabalho tem como foco central a historiografia brasileira da ciência, voltandose especificamente para a vida e obra de um matemático-físico da contemporaneidade e passa a fazer parte de uma série de pesquisas que investigam, organizam e descrevem itinerários pessoais, intelectuais e profissionais de cientistas e educadores brasileiros.

Este trabalho tem como objetivo reescrever e apreentar a história de vida, formação acadêmica, atuação profissional e produção intelectual do multifacetado cientista Guilherme Mauricio Souza Marcos de La Penha.

\section{Faces de Guilherme de La Penha}

Com base nos apontamentos de trabalhos publicados, apresentamos uma síntese dos diversos caminhos percorridos por Guilherme De La Penha de modo à carcterizar as diversas faces deste cientista. Observamos inicialmente as diversas contribuições científicas em inúmeros trabalhos acadêmicos, dentre eles, destacam-se os trabalhos relacionados a Leonard Euler, bem como suas contribuições nas outras áreas de atuação a exemplo da gestão e como professor.

\section{Nos passos de Leonhar Euler}

Guilherme de La Penha escreveu diversos artigos sobre Leonhard Euler, no período que compreende os anos de 1982 a 1986, publicados na Revista do Professor de Matemática, Revista Humanidades, Monografias da Sociedade Paranaense de Matemática, Revista Perspicillum e Museu Paraense Emílio Goeldi.

Dentre essas obras, destacam-se quatro artigos, bastante robustos e ricos em detalhes sobre a vida e produção de Euler, escritos ao longo do ano de 1983 no Brasil. Estes quatro artigos foram enviados à Embaixada Suiça no Brasil por La Penha e por meio de correspondência oficial ofício o governo suíço apresenta seus agradecimentos tendo em vista as comemorações do bicentenário de morte de Euler. Os artigos encaminhados foram: Éloge de Euler (1983); $e^{\square . i}+1=0$ ou Leonardo Euler (1983); Euler: Mestre da Matemática, expoente científico do Iluminismo (1983) e Nas cartas a uma Princesa da Alemanha, a lógica dedutiva como prólogo a filosofia de Euler (1983). 
Com base nos artigos sobre Euler percebemos que La Penha exalta o nome de Euler como aquele intelectual que se alinhará sempre entre os que ocupam os primeiros lugares na Matemática. Decorre das análises dos doze artigos que La Penha tinha Euler como um ídolo, fato que nos leva a admitir a possibilidade de Euler ter sido o principal influenciador da obra e do modelo de cientísta adotado La Penha.

Sintetizamos os artigos escritos por La Penha sobre Euler no Quadro a seguir:

Quadro 3 - Euler no prisma de Guilherme de La Penha

\begin{tabular}{|c|c|c|}
\hline Ano & Obra & Publicado em: \\
\hline 1982 & A grandeza do desconhecido Euler & Revista Humanidades \\
\hline 1983 & $\begin{array}{c}\text { Editorial da Revista do Professor de } \\
\text { Matemática }\end{array}$ & $\mathrm{RPM} \mathrm{n}^{\circ} 3$ \\
\hline 1983 & Leonhard Euler & RPM n 3 \\
\hline 1983 & Euler e a Topologia & $\mathrm{RPM} \mathrm{n}^{\circ} 3$ \\
\hline 1983 & $e^{\square . i}+1=0$ ou Leonardo Euler & MPEG \\
\hline 1983 & Éloge de Euler & LNCC \\
\hline 1983 & $\begin{array}{l}\text { Euler: Mestre da Matemática, expoente } \\
\text { científico do Iluminismo }\end{array}$ & MPEG \\
\hline 1984 & Euler e a Teoria dos Números & $\mathrm{RPM}^{\circ} 4$ \\
\hline 1984 & $\begin{array}{c}\text { Nas cartas a uma Princesa da Alemanha, a } \\
\text { lógica dedutiva como prólogo a filosofia de } \\
\text { Euler }\end{array}$ & $\begin{array}{l}\text { Monografias da Sociedade } \\
\text { Paranaense de Matemática }\end{array}$ \\
\hline 1986 & $\begin{array}{l}\text { Euler: Da Filosofia Natural a Expoente } \\
\text { Científico do Iluminismo }\end{array}$ & Revista Perspicillum \\
\hline 1986 & A Evolução do Conceito de Função & MPEG \\
\hline 1986 & $\begin{array}{c}\text { Resenha e ensaio histórico sobre a álgebra de } \\
\text { Euler }\end{array}$ & MPEG \\
\hline
\end{tabular}


Fonte: Chaquiam (2012)

De acordo com Chaquiam (2012), no livro Writing the History of Mathematics: Its Historical Development, capítulo 15, 15.2 América do Sul, 15.2.1 Observações historiográficas, página 254, Ubiratan D’Ambrósio tece o seguinte comentário sobre La Penha: Also, GUILHERME M. DE LA PENHA (1943-1996) studied Euler, mainly analysing the Lettres à une princesse d'Allemagne (Letters to a German Princess), ou seja, La Penha foi um estudioso da obra de Euler, principalmente as Cartas a uma Princesa Alemã.

No artigo A grandeza do desconhecido Euler, publicado na Revista Humanidades em 1982, La Penha reconhece que grande parte dos seus conhecimentos sobre Euler são devidos à orientação e aos escritos de C. Truesdell, inicialmente apresentados por Alberto Coimbra por volta de 1963.

\section{Aspectos relacionados à gestão}

La Penha ocupou cargos como gestor acadêmico e público, atuando em Universidades, Institutos, Fundações e Autarquias, além de cargos voltados para área da educação e, em alguns momentos, prestou serviço em Secretarias e Museus, dentre outros cargos supracidados a seguir.

La Penha ocupou cargos tais como: Vice-presidente do Conselho Nacional de Desenvolvimento Cientifico e Tecnológico, vinculado a Secretaria de Planejamento da Presidência da República e, nessa qualidade, foi Vice-Presidente do Conselho Cientifico e Tecnológico do CNPq. Além disso, foi Supervisor do Observatório Nacional do Rio de Janeiro, São Paulo, Vassouras, Itajubá e Tatuoca; atuaou no Instituto de Pesquisas Espaciais em São José dos Campos; no Instituto Nacional de Pesquisas da Amazônia em Manaus, Rio Branco e Tucuruí; do Museu Paraense Emílio Goeldi (MPEG) em Belém e Carajás; no Instituto de Matemática Pura e Aplicada do Rio de Janeiro; no Laboratório de Computação Cientifica (LNCC) no Rio de Janeiro e do Centro Brasileiro de Pesquisas Físicas no Rio de Janeiro. Em 1983, de volta à Belém, foi nomeado Assistente Especial do CNPq para a Amazônia, passando a atuar no MPEG, restaurando a Biblioteca do MPEG e a Biblioteca Pública da Pará. 
Após ocupar o cargo de Secretário de Ciência e Tecnologia na Organização dos Estados Americanos (OEA), atendeu aos pedidos de retorno e, em agosto 1985, La Penha retorna a Belém para dirigir o MPEG, ocupando também o cargo de Secretário de Cultura do Estado do Pará. Em 1987 deixou o cargo de Secretário para atuar como Assessor Especial do Governador Hélio da Mota Gueiros, dedicando-se a estruturação e implantação da Secretária de Estado da Ciência, Tecnologia e Meio Ambiente do Pará, até que, em 1991, deixou a direção do MPEG, concretizando importantes obras cientificas para o Estado como a Estação de Pesquisas "Ferreira Penna" na floresta de Caixiuanã, em Melgaço, no Pará, iniciada em 1990 e concluída em 1992, na gestão do químico Guilherme Maia.

No período de 1991 a 1994 foi Secretário de Cultura do Pará e Presidente da Fundação Cultural Tancredo Neves, durante o governo de Jader Barbalho, período em que criou o Salão Paraense de Artes Plásticas, instalou o Museu do Estado do Pará e preparou o inventário de todos os bens artísticos e culturais de Belém. Na qualidade de Secretário de Cultura ordenou a publicação das obras completas do escritor paraense Bruno de Menezes, publicação agraciada com o Prêmio Jabuti em 1994.

Antes de completar 54 anos, no dia 6 de fevereiro de 1996 veio a falecer, quando ocupava o cargo de Diretor de Programas Espaciais da Secretária de Assuntos Estratégicos da Agência Espacial Brasileira, tornando-se uma grande perda para a vida acadêmica e administrativa de ciência no Brasil.

Considerando a atuação de La Penha nos diversos cargos ocupados, podemos concluir que foi um gestor preocupado com a organização, atualização e consolidação das instituições, tanto do ponto de vista da ciência, quanto da tecnologia. Além disso, embora tenha ocupado diversos cargos importantes voltados para a gestão, La Penha também dedicava seu tempo para as produções cientificas:

Publicava artigos voltados ao currículo e ao ensino de matemática, evidenciando sua preocupação, na época, com a reformulação curricular de modo a disseminar mais adequadamente a matemática a estudantes de outras ciências visando à conscientização da necessidade de mudanças no ensino da matemática em conjunto com as outras profissões tais como: Algumas consequiências para a Matemática do Ensino Superior em Ciências Aplicadas, publicado na Revista EDUCAÇÃO, V. 11, Brasília, 1974, 93 -

100 p; Como estudar matemática? (Chaquiam, 2012) 


\section{Produções científicas}

Ao longo de sua carreira produziu diversos artigos publicados em periódicos especializados nacionais e internacionais. Foi Editor e traduziu três livros texto de circulação internacional. Teve trabalhos científicos citados em diversas publicações internacionais, em especial no Handbuch der Physik, Springer Verlag, Heidelberg.

O livro Introdução a Álgebra Linear, escrito em perceria com Mina S. de Carakushansky em 1977, foi baseado nas experiências em ministrar e coordenar o ensino das disciplinas Álgebra Linear I e II, no ciclo básico universitário.

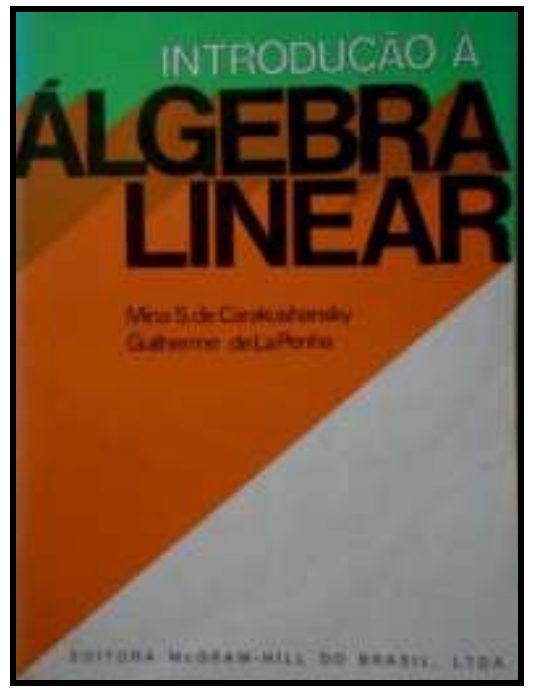

Figura 1: Capa original do livro

Fonte: Acervo pessoal dos autores

De acordo com La Penha e Mina Carakushansky o livro Autovalores $e$ Diagonalização (1976) complementam o livro Introdução à Álgebra Linear dos mesmos autores e iniciam àqueles que desejam enveredar pela teoria dos autovalores.

O livro Espaço Vetorial de Dimensão Finita é uma tradução do original que La Penha considerava o clássico dos clássicos sobre o assunto. 


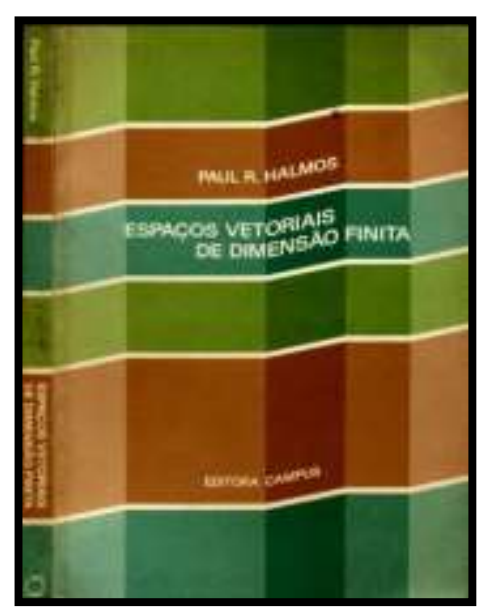

Figura 2: Capa do livro traduzido

Fonte: Acervo pessoal dos autores

O livro Introdução ao Método de Riemann em Problemas de Contorno do Tipo Hiperbólico a Duas Variáveis (1976), de acordo com La Penha, o conteúdo do texto teve como base suas notas de aula dos cursos ministrados durante o mês de julho de 1967, na Universidade de Houston, e, em janeiro de 1970 (escola de verão), na Universidade Federal do Rio de Janeiro.

De acordo com Chaquiam (2012), um importante trabalho elaborado por La Penha em parceria com Morton E. Gurtin, foi On the Termodynamics of Mixtures - I. Mixtures of Rigid Heat Conductors" faz parte do "Archive Rational Mechanics and Analysis, volume 36, número 5

Num de seus artigos La Penha destaca que o conceito de função real, embora de início, como seus predecessores, tivesse usado um conceito muito mais restrito e vago, suas próprias descobertas na teoria das equações, das derivadas e da propagação de onda mostraram-lhe o modo claro de introduzir o conceito de função da maneira que todo matemático vem fazendo desde 1837.

\section{Contribuições científicas}

Em entrevista com Chaquiam (2012) o professor Luis Adauto afirma que o International Symposium on Continuum Mechanics and Partial Differential Equations, realizado no período de 01 a 05 de agosto de 1977, no Instituto de Matemática da UFRJ, foi mais uma importante contribuição de La Penha. Este evento foi previsto com o objetivo de familiarizar os matemáticos e outros cientistas da física e da engenharia com as principais linhas de pesquisa moderna em assuntos interrelacionados, fato que permitiu 
estimular discussões que levaram à investigação de novos problemas ou retomar os antigos ainda em aberto.

O professor José Maria Filardo Bassalo afirmou numa das entrevistas concedidas a Chaquiam (2012) que foi durante o período em que La Penha esteve a frente da FINEP que teve oportunidade de exercer o papel de agente-gerenciador da pesquisa científica ao nível nacional, e mais, nesse período que La Penha voltou à Belém, a convite do Departamento de Física da UFPA, para organizar o primeiro Curso de Especialização promovido por esse Departamento e doou a esse Departamento a maior parte da sua biblioteca especializada em Matemática.

De acordo com Chaquiam (2012), na qualidade de vice-presidente do CNPq, La Penha mostrava-se preocupado com o processo acelerado de ocupação da Amazônia no artigo intitulado O papel da pesquisa para uma ocupação racional da Amazônia, aonde destaca que o papel da ciência e da tecnologia na Amazônia é o de solucionar as contradições pela abertura de novos caminhos que possibilitem harmonizar: retorno econômico versus proteção ambiental; migração populacional versus proteção das populações primiticas; qualidade de vida versus desbravar áreas sem mínima estrutura.

La Penha organizou Seminário Internacional sobre Meio Ambiente, Pobreza e Desenvolvimento da Amazônia - SIMDAMAZÔNIA - O não da Amazônia, no período de 16 a 19 de fevereiro de 1992, com apoio da ONU/UNICEF e do Governo do Estado do Pará. Neste evento foram debatidos 10 temas, sendo o Tema 6, coordenado por La Penha, abordou $O$ papel das instituições governamentais e não governamentais de ciência, tecnologia e desenvolvimento na questão ambiental.

No livro O Museu Paraense Emílio Goeldi (1986), La Penha menciona a importância de se publicar coleções científicas como as existentes no MPEG, que, de certo modo, homenageia os idealistas que o conceberam, cientistas e funcionários que ao longo dos anos vêm labutando, em condições na maioria das vezes adversas.

\section{O poliglota}

Das pesquisas em livrarias virtuais resultaram a aquisição de livros e revistas com publicações de La Penha, outros, na qualidade de revisor técnico ou tradudor, por exemplo, o primeiro artigo científico publicado como professor da recém criada 
Universidade Federal de Juiz de Fora, em 1964, na revista Lumina Spargere; segundo o próprio, o livro Elementos de Mecânica Racional Clássica, resultante do $9^{\circ}$ Colóquio Brasileiro de Matemática, realizado R. Halmos, traduzido por La Penha e editado em 1978 pela editora Campus; o livro Introdução à Matemática para Biocientistas, de E. Batschelet, traduzido para o português sob em Poços de Caldas, em julho de 1973; o livro Espaços Vetoriais de Dimensão Finita, de Paul a revisão técnica de La Penha e o Boletim da Sociedade Brasileira de Matemática Aplicada e Computacional, ano III, nº 3, de 1982, com a conferência de abertura do V Congresso Nacional de Matemática Aplicada e Computacional, intitulada "Sobre as Histórias da História da Mecânica do Século XVIII `. Por ele ser poliglota, La penha também realizava trabalhos como tradutor de livros para o portuglêns e bem como as revisões técnica.

\section{Sua relação com a Física}

Após estudos exploratórios e empíricos em acervos, onde consta que a produção acadêmica de Guilherme de La Penha é a prova viva de sua dedicação, seriedade e visão futura de educador, matemático-físico membro de diversas sociedades científicas.

Embora sua formação básica seja em Engenharia Mecânica, obtida na PUC-Rio em 1964, nota-se a sua forte relação com matemática, observado nos trabalhos desenvolvidos durante o curso de especialização no IMPA e, posteriormente, os diversos textos voltados a mesma, neste sentido La Penha é considerado por diversos professores um matemático-fisíco e não um físico-matemático como muitos pensavam antes.

\section{Escreve sobre música e publica usando pseudônimo}

Considerado por muitos um visionário, um poeta, detentor de uma das mentes mais brilhantes, principalmente pelos artigos publicados nas mais diversas áreas do conhecimento, incluindo-se aí, os artigos publicados sobre poesias e músicas clássicas.

A partir do exposto anteriormente nota-se a importância do teórico no meio acadêmico. Assim, é possível assegurar que La Penha tinha um perfil intelectual que pode ser considerado um intelectual transdisciplinar que defende a possibilidade de se formar um cientista uno e múltiplo, de atitude não linear e que dialoga com todas outras áreas de modo a ser compreendido sob um modelo de cientista neo-iluminista para o século XXI. 


\section{Considerações finais}

Este trabalho atingiu o objetivo de reescrever a história de vida, formação acadêmica, atuação profissional e produção intelectual do cientista de Guilherme Mauricio Souza Marcos de La Penha, sob diversas facetas, tendo em vista apresentá-lo à comunidade acadêmica.

Com base nos apontamentos apresentados, destacamos as convergências argumentativas que validam nossos objetivos norteadores anunciados nas páginas iniciais deste trabalho, ou seja, argumentamos favoravelmente sob o perfil de um intelectual múltiplo, cujo pensamento sobre ciência, tecnologia, formação de cientistas e educadores estiveram em harmonia nos seus escritos e na sua prática profissional, caracterizando como um cientista multifacetado.

Reiteramos que alguns aspectos ficaram bem identificados no decorrer deste trabalho, como por exemplo, as insistências de La Penha em defender o perfil de um cientista que a universidade deveria formar; um cientista preparado para se incluir no mercado de trabalho no fim do século XX e no início do século XXI; um cientista que exerce a pesquisa, a gestão e a administração, bem como se constituísse em um educador comprometido com esse processo formativo e formador. Para La Penha, o cientista não deve apenas produzir conhecimento, mas deve também gerenciar, ser líder e estar à frente do seu tempo.

Destacamos que ele desenvolveu um corpo significativo de trabalhos ligados à cultura, a literatura com interfaces múltiplas sem perder o fio condutor de sua área de pesquisa, caracterizando-se sempre como um cientista transdisciplinar.

Os artigos de La Penha sobre Euler demonstram que era profundo conhecedor da obra desse magnífico matemático, principalmente pela forma abrangente e profunda com que tratava o legado de Euler, de certa forma, até certo ponto romântica.

A constituição da Seção Guilherme de La Penha garante a preservação de um patrimônio científico e a futura mostra Guilherme de La Penha que está sendo idealizada em parcerias com os setores do MPEG.

Em Chaquiam (2012) observa-se que La Penha foi um homem inquieto por natureza, perseverante na busca de um ideal, incompreendido por alguns, impaciente com as comodidades e marasmos funcionais, amado por sua família e por aqueles que reconhecem a importância do trabalho desenvolvido por ele. 
Neste trabalho foi possível entrelaçar alguns poucos elementos da vida, da formação acadêmica e atuação profissional de Guilherme de La Penha com elementos históricos relacionados à sua atuação em diversos setores acadêmicos e de administração pública e científica, demonstrando que foi um cientista multifacetado além de ser um incentivador da divulgação e comunicação dos conhecimentos produzidos no âmbito da cultura, da ciência, da educação e da tecnologia.

\section{Referências}

BASSALO, José Maria Filardo. La Penha: Gerador e Gerenciador da Ciência. Centro Brasileiro de Pesquisas Físicas. Revista Ciência e Sociedade. v. 14. Rio de Janeiro, 1997.

CHAQUiAM, M.; SILVA, E. O. C. Alguns passos de Guilherme de La Penha no Brasil e no exterior. Anais do VIII Seminário Nacional de História da Matemática. SBHMat: Belém, 2009.

CHAQUIAM, M.; MENDES, I. A. A face acadêmica de Guilherme de La Penha. Anais IV Congresso Internacional de Pesquisa (Auto)biográfica. São Paulo (SP): USP, 2010.

CHAQUIAM, M.; MENDES, I. A. Guilherme de La Penha - cientista paraense do século XX. Anais do V Colóquio de História e Tecnologia no Ensino da Matemática. Recife: UFPE, 2010.

CHAQUIAM, M.; MENDES, I. A. A visão de La Penha sobre Euler. Anais do Encontro Brasileiro de Estudantes de Pós-Graduação - EBRAPEM. Campo Grande (MT): UFMS, 2010.

CHAQUIAM, M.; MENDES, I. A. GUILHERME DE LA PENHA - Um cientista no obscurantismo. X Encontro Nacional de Educação Matemática. Salvador - BA, 2010.

CHAQUIAM, M.; MENDES, I. A. Do inventário a idealização da mostra Guilherme de La Penha. Anais da XIII Conferência Interamericana de Educação Matemática. Recife (PE), CIAEM, 2011.

CHAQUIAM, Miguel. Guilherme de La Penha: uma história do seu itinerário intelectual em três dimensões. 2012. 285 f. Tese (Doutorado em Educação) - Universidade Federal do Rio Grande do Norte, Natal, 2012.

CHAQUIAM, M.; MENDES, I. A. Produção Intelectual de Guilherme de La Penha. Anais do Seminário Nacional de História da Matemática. Campinas (SP), SBHMat, 2013. 
CHAQUiAM, M.; MENDES, I. A. Guilherme de La Penha e a Amazônia. Anais Eletrônicos do $14^{\circ}$ Seminário Nacional de História da Ciência e da Tecnologia. Belo Horizonte, Campus Pampulha da Universidade Federal de Minas Gerais - UFMG, 2014. 